\title{
Noncoherent imagery: a novel approach
}

\author{
J. Ojeda-Castañeda* and C. M. Gómez-Sarabia \\ Electronics Department, University of Guanajuato, Salamanca, 36885, Guanajuato, México
}

Received November 15, 2010; accepted November 27, 2010; published December 31, 2010

\begin{abstract}
We use an orthonormal expansion for relating the optical transfer function with the expectation values, as defined in quantum physics. A certain set of orthonormal functions is useful for writing the output irradiance distribution as a quasi Gabor expansion of the input irradiance distribution. The coefficients of this later expansion are cross correlations measurements of similarity. We show that the expectation values are related to the cross correlations coefficients by a discrete Fourier transform.
\end{abstract}

\section{Introduction}

For mobile telephony and for imaging in computer assisted activities, it is desirable to gather pictures, using low price devices, with nonconventional optical elements and detectors interconnected to digital image processors.

Due to this interconnectivity, new optical devices have been proposed for extending the depth of field, while preserving the full pupil aperture [1]-[10]. These new devices are able to generate modulation transfers functions (MTFs) with the following two features. The MTFs have a low sensitivity to focus errors. And the MTFs do not have zero values inside their passband. Hence, simple digital algorithms can be applied to restore digitally any visibility loss in the MTF.

Here we explore some analytical tools for gaining physical insight when designing these new optical elements.

In section 2, we explore the use of orthonormal expansions for relating the OTF with the expectation values, as defined in quantum physics [11]. Then, in section 3, we identify a suitable set of orthonormal functions that relate the output irradiance distribution as a quasi Gabor expansion of the input irradiance distribution [12]. We indicate that the coefficients of the quasi Gabor expansion are similarity cross correlation coefficients [13]. In section 4, we show that the cross correlation coefficients are related to the expectation values by a discrete Fourier transform. For the sake of clarity our discussion is 1-D; and we assume that the imaging process has a unit magnification.

*E-mail: jorge_ojedacastaneda@yahoo.com

\section{Orthonormal expansions}

The input irradiance distribution is suitably represented as the following expansion

$$
I_{0}(x)=\sum_{s=-\infty}^{\infty} a_{s} \phi_{s}(x)
$$

In Eq. (1) the lower case Greek letter phi denotes the s-th component of a suitable set of orthonormal function, as common in mathematical physics. See Appendix A. The Fourier transform of Eq. (1), here denoted as the Fourier spectrum of the input, is

$$
\begin{aligned}
\tilde{I}_{0}(\mu) & =\int_{-\infty}^{\infty} I_{0}(x) \exp (-i 2 \pi \mu x) d x \\
& =\sum_{s=-\infty}^{\infty} a_{s} \Phi_{s}(\mu) .
\end{aligned}
$$

The function phi (in upper case Greek letter) denotes the Fourier transform of the function phi in lower case characters. Next, we express the Fourier spectrum of the output as

$$
\begin{aligned}
\tilde{I}(\mu ; W) & =H(\mu ; W) \tilde{I}_{0}(\mu) \\
& =\sum_{s=-\infty}^{\infty} a_{s} H(\mu ; W) \Phi_{s}(\mu) .
\end{aligned}
$$

In Eq. (3) we denote as $H(\mu ; W)$ the optical transfer function (OTF) of the optical system. In a symbolic fashion, the Roman letter $\mathrm{W}$ represents the influence of the wave aberrations on the OTF. Of course, the MTF is the modulus of the OTF. As is indicated in Appendix A, the functions $\Phi_{s}(\mu)$ also form an orthonormal set.

Now, we propose to express $H(\mu ; W) \Phi_{s}(\mu)$ in terms of the initial set of orthonormal function. That is,

$$
H(\mu ; W) \Phi_{s}(\mu)=\sum_{m=-\infty}^{\infty}{ }_{s} C_{m}(W) \Phi_{m}(\mu)
$$


we note that, as it is common when using an orthonormal base, the new coefficients are obtained by evaluating

$$
{ }_{m} C_{s}(W)=\int_{-\infty}^{\infty} \Phi_{m} *(\mu) H(\mu ; W) \Phi_{s}(\mu) d \mu .
$$

We recognize that the new coefficients are obtained by performing a mathematical operation that is similar to the expectation value, as used in quantum physics:

$$
{ }_{m} C_{s}(W)=\langle m|H(\mu ; W)| s\rangle .
$$

Now, by substituting Eq. (4) in Eq. (3), we have that the Fourier spectrum of the output is

$$
\tilde{I}(\mu ; W)=\sum_{m=-\infty}^{\infty}\left\{\sum_{s=-\infty}^{\infty}\langle m|H(\mu ; W)| s\rangle a_{s}\right\} \quad \Phi_{m}(\mu) .
$$

Finally, for obtaining the irradiance distribution at the output, we take the inverse Fourier transform of Eq. (6). That is,

$$
I(x ; W)=\sum_{m=-\infty}^{\infty}\left\{\sum_{s=-\infty}^{\infty}\langle m|H(\mu ; W)| s\rangle a_{s}\right\} \quad \phi_{m}(x) .
$$

We note that the expression in Eq. (7) describes the output irradiance distribution using the same set of orthonormal functions that are used for representing the input irradiance distribution. Of course, our result is valid for any set of orthonormal functions. However, as we discuss next, one set of orthonormal functions is particularly useful.

\section{Quasi Gabor expansion}

For testing the performance of an optical system, working under noncoherent illumination, it is common to use as input a periodic test pattern. Hence, as schematically shown in Fig. 1, we assume that at the input plane the irradiance distribution is a binary grating, with period $d$, and with duty cycle (or fill factor) equal to $d / N$, where $\mathrm{N}$ is any natural number. This input binary pattern can be represented by the Fourier series

$$
\phi_{0}(x)=\left(\sqrt{N / d} \sum_{s=-\infty}^{\infty}[\sin c(s / N)] \exp (i 2 \pi x s / d) .\right.
$$

Next, by taking advantage of the binary nature of the input, we define the following set of $\mathrm{N}$ orthonormal functions

$$
\phi_{n}(x)=\phi_{0}(x-n d / N)
$$

By construction, we know that

$$
\int_{-d / 2}^{d / 2} \phi_{m} *(x) \phi_{n}(x) d x=\delta_{m \cdot n}
$$

In Eq. (10) we use the symbol $\delta_{m . n}$ for representing a Kronecker delta, of any two natural numbers $\mathrm{m}$ and $\mathrm{n}$ such that $\mathrm{m}$ and $\mathrm{n}$ are smaller than $\mathrm{N}$.

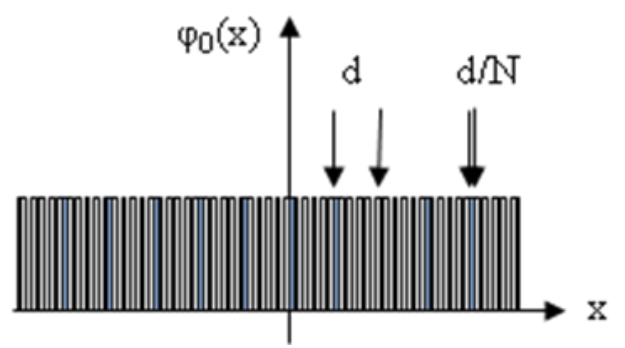

Fig. 1. Binary grating with period $d$, and with duty cycle $\mathrm{d} / \mathrm{N}$.

Now, for testing the performance of the optical system, we consider the input irradiance distribution to be $I_{0}(x)=\varphi_{0}(x)$.

Next, we suggest expressing the output irradiance distribution as the following quasi Gabor expansion

$$
\begin{aligned}
I(x ; W) & =\sum_{n=0}^{N-1} \sigma_{n}(W) \phi_{n}(x) \\
& =\sum_{n=0}^{N-1} \sigma_{n}(W) I_{0}(x-n d / N) .
\end{aligned}
$$

The coefficients $\sigma_{n}(W)$ are obtained by evaluating the cross correlation

$$
\sigma_{p}(W)=(N / d) \int_{-d / 2}^{d / 2} I(x+p d / N ; W) I_{o}(x) d x
$$

The mathematical expression in Eq. (12) is similar to the definition of Fresnel similarity in Ref. [13], if W stands for a focus error. Hence, one can consider that the cross coefficients measure the resemblance between the input irradiance distribution and the output irradiance distribution, if the optical system suffers from aberrations. In what follows, we relate the expectation values with the cross correlation coefficients. 


\section{Discrete Fourier transformation}

If we substitute Eq. (8) in Eq. (11), we have that the output irradiance distribution can be written as

$$
\begin{aligned}
& I(x ; W)= \sum_{n=0}^{N-1} \sigma_{n}(W) I_{0}(x-n d / N) \\
&=\sum_{m=-M}^{M}\left\{\sum_{n=0}^{N-1} \sigma_{n}(W) \exp (-i 2 \pi m n / N)\right\} \\
& \operatorname{sinc}(m / N) \exp (i 2 \pi x m / d) .
\end{aligned}
$$

We recognize that Eq. (13) is a possible representation of Eq. (7) when the orthonormal set is

$$
\phi_{m}(x)=\sqrt{(1 / d)} \quad \exp (i 2 \pi m x / d)
$$

and

$$
a_{m}=\operatorname{sinc}(m / N)
$$

Hence, from a simple comparison of Eqs (7) and (13), we conclude that

$$
\langle m|H(\mu ; W)| m\rangle .=\sum_{n=0}^{N-1} \sigma_{n}(W) \exp (-i 2 \pi m n / N) .
$$

Or equivalently,

$$
\sigma_{n}(W)=(1 / N) \sum_{m=0}^{N-1}\langle m|H| m\rangle \exp (i 2 \pi n m / N) .
$$

Therefore, when wave aberrations are present, the expectation values of the OTF can be understood as the Fourier spectrum of the cross correlation coefficients, which measure the similarity between the input and the output. Thus, the cross correlation coefficients can be used as image quality criteria for evaluating the impact of wave aberrations on the OTF.

\section{Concluding remarks}

We have shown that by using an orthonormal set of functions, one can link the mathematical methods for evaluating the expectation values in quantum physics, with the analysis of the impact of wave aberrations on the OTF. We have identified one set of orthonormal functions that helps to express the output irradiance distribution as a quasi Gabor expansion of the input irradiance distribution. The coefficients of this latter expansion are cross correlation values that measure the similarity between the input and the output. Furthermore, we have shown that the expectation values of the OTF and the cross correlation coefficients are related to each other by a discrete Fourier transform. Therefore, the cross correlation coefficients may be useful as image quality criteria, when wave aberrations are present.

We gratefully acknowledge the financial support of PROMEP, SNI and the University of Guanajuato, México.

\section{Appendix $A$}

It is not so well known that if one has a set of functions, $\phi_{m}(x)$, then their Fourier transform, $\Phi_{m}(\mu)$, also form a orthonormal set, as we show next. By definition

$$
\Phi_{m}(\mu)=\int_{-\infty}^{\infty} \phi_{m}(x) \exp (-i 2 \pi \mu x) d x
$$

Then,

$$
\begin{aligned}
\int_{-\infty}^{\infty} \Phi_{m} *(\mu) \Phi_{n}(\mu) d \mu & =\int_{-\infty}^{\infty} \int_{-\infty}^{\infty} \int_{-\infty}^{\infty} \phi_{m}^{*}(x) \phi_{n}(y) \\
& =\int_{-\infty}^{\infty} \int_{-\infty}^{\infty} \phi_{m} *(x) \phi_{n}(y) \delta(x-y) d x d y \\
& =\int_{-\infty}^{\infty} \phi_{m} *(x) \phi_{n}(x) d x \\
& =\delta_{m, n} .
\end{aligned}
$$

Along the mathematical development of equation (A2), we identify and use the Dirac delta $\delta(x-y)$, for proving that both $\phi_{m}(x)$ and $\Phi_{m}(\mu)$ form a set of orthonormal functions.

\section{References}

[1] J. Ojeda-Castañeda, R. Ramos, A. Noyola-Isgleas, Appl. Opt. 27, 2583 (1988).

[2] J. Ojeda-Castañeda, L.R. Berriel-Valdos Ramos, E. Montes, Appl. Opt. 27, 790 (1988)

[3] E.R. Dowski, Jr., W.T. Cathey, Appl. Opt. 34, 1859 (1995).

[4] W. Chi, N. George, Opt. Lett. 26, 875 (2001).

[5] S. Mezouari and A.A. Harvey, Opt. Lett. 28, 771 (2003).

[6] A. Sauceda, J. Ojeda-Castañeda, Opt. Lett. 29, 560 (2004).

[7] A. Castro, J. Ojeda-Castañeda, Appl. Opt. 43, 3474 (2004).

[8] E. Ben-Eliezer, E. Maron, N. Konforti, Z. Zalevsky, Appl. Opt. 44, 2792 (2005).

[9] Y. Takahashi, S. Komatsu, Opt. Lett. 33, 1515 (2008)

[10] J. Ojeda-Castañeda, J.E.A. Landgrave, C.M. Gómez-Sarabia, Appl. Opt. 47, E99 (2008).

[11] A.A. Sokolov, I.M. Ternov, V.Ch. Zhukovskii, Quantum mechanics (URCC Publishers, Moscow 1994).

[12] M.J. Bastiaans, Signal and Image Representation in Combined Spaces (Academic Press, 1995).

[13] A.W. Lohmann, J. Ojeda-Castañeda, Opt. Comm. 249, 397 (2005). 